\title{
Prospects for High Angular Resolution Instrumentation at Millimeter and Submillimeter Wavelengths
}

\author{
Karl M. Menten \\ Max-Planck-Institut für Radioastronomie, Auf dem Hügel 69, D-53121 \\ Bonn, Germany
}

\begin{abstract}
Millimeter- and submillimeter wavelength interferometry is a powerful technique allowing imaging of dust and molecules in a multitude of astronomical environments. With the arrival of the Atacama Large Millimeter Array (ALMA), such studies will be possible with unprecedented sensitivity and a spatial resolution similar to the diffraction limit of large optical/infrared telescopes. In this paper, we mention a few aspects of (sub)millimeter astronomy and provide a short summary of ALMA's capabilities.
\end{abstract}

\section{High Resolution Studies of Dust and Molecules in the Universe}

At millimeter and submillimeter wavelengths, the broad band emission from the interstellar medium of the Milky Way and external galaxies is dominated by thermal grey body radiation from cold and warm dust grains. For "normal" spirals, far-infrared dust emission accounts for a significant part of the total luminosity (de Jong et al. 1984; Braine \& Hughes 1999) and completely dominates the energy output of ultraluminous infrared galaxies (ULIRGS, see Sanders \& Mirabel 1996).

In the Rayleigh-Jeans regime, the flux density, $S_{\nu}$, of a dust source at frequency $\nu$ is proportional to $\nu^{2+\beta}$, with $1<\beta<2$. Because of this and the increasing absorption by the Earth's atmosphere toward higher frequencies, one finds that from ground-based observatories dust emission is optimally detected between frequencies of 200 and $360 \mathrm{GHz}$, corresponding to wavelengths, $\lambda$, of 1.5 and $0.83 \mathrm{~mm}$, although the spectral energy distribution of the total dust emission from most galaxies peaks in the far-infrared (around $3 \mathrm{THz}$ or 100 $\mu \mathrm{m})$.

Most interestingly, for high redshifts, $z$, the dust emission peak shifts from the far-infrared to submillimeter and millimeter wavelengths. The resulting enhancement of the (sub)millimeter emission (over)compensates the dimming due to the source's distance (see, e.g., Franceschini et al. 1991; Blain \& Longair 1993). This "negative $K$ correction" allows detection of dust heated by massive starbursts in ultraluminous infrared galaxies at $z>4$ (see Fig. 1). Such observations provide unique information on contributions to the global star formation history of the universe that are missed by optical and even (near)infrared surveys due to strong extinction (Hughes et al. 1998). 


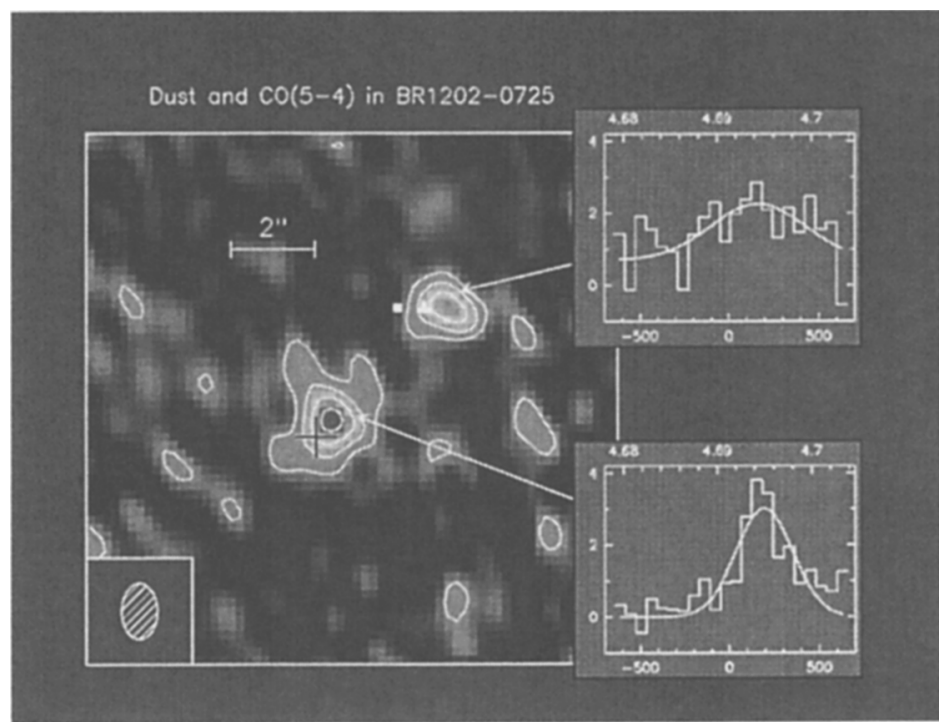

Figure 1. Image of the $1.3 \mathrm{~mm}$ dust continuum emission toward the quasar BR1202-07 at $z=4.69$ produced with the IRAM interferometer. The insets show the CO $J=5-4$ spectra of each component (see Omont et al. 1996).

In addition to dust emission, (sub)mm molecular lines are powerful probes for an extremely wide range of astrophysical environments: from cold molecular clouds at a temperature, $T$, of $10 \mathrm{~K}$ and with a molecular hydrogen $\left(\mathrm{H}_{2}\right)$ density, $n_{\mathrm{H}_{2}}$, of $10^{2} \mathrm{~cm}^{-3}$ to the innermost parts of the circumstellar envelopes around red giant stars, where $T \approx 1500 \mathrm{~K}$ and $n_{\mathrm{H}_{2}} \approx 10^{10} \mathrm{~cm}^{-3}$. In particular, the carbon monoxide (CO) molecule, whose rotational lines are observed in all of these environments, is widely used to map the global molecular content of the Milky Way and external galaxies as a tracer of the $10^{4}$ times more abundant, but difficult to observe, $\mathrm{H}_{2}$. High redshift $\mathrm{CO}$ emission has been detected toward $\approx 10$ sources with redshifts between 2.3 and 4.7 (see Fig. 1 and Barvainis 1999).

Since (sub)millimeter single dish telescopes with diffraction-limited resolutions better than $\approx 10^{\prime \prime}$ become prohibitively expensive to build, achieving higher spatial resolution requires the use of interferometric techniques. Today, a total of four millimeter-wavelength interferometer arrays are working highly successfully at frequencies between 80 and $270 \mathrm{GHz}(3.8 \mathrm{~mm}>\lambda>1.1 \mathrm{~mm})$, reaching resolutions $\lesssim 0$ " 5 . These are the Hat Creek Array operated by the Berkeley-Illinois-Maryland consortium, the Owens Valley Millimeter Array operated by Caltech, the Nobeyama Millimeter Array of the National Astronomical Observatory of Japan, and the interferometer operated by the Institute for Radio Astronomy at Millimeter Wavelengths (IRAM) on the Plateau de Bure in the French Alps. The IRAM instrument is the one with the highest sensitivity for point-like sources. The first multi-element submillimeter interferometer, the 


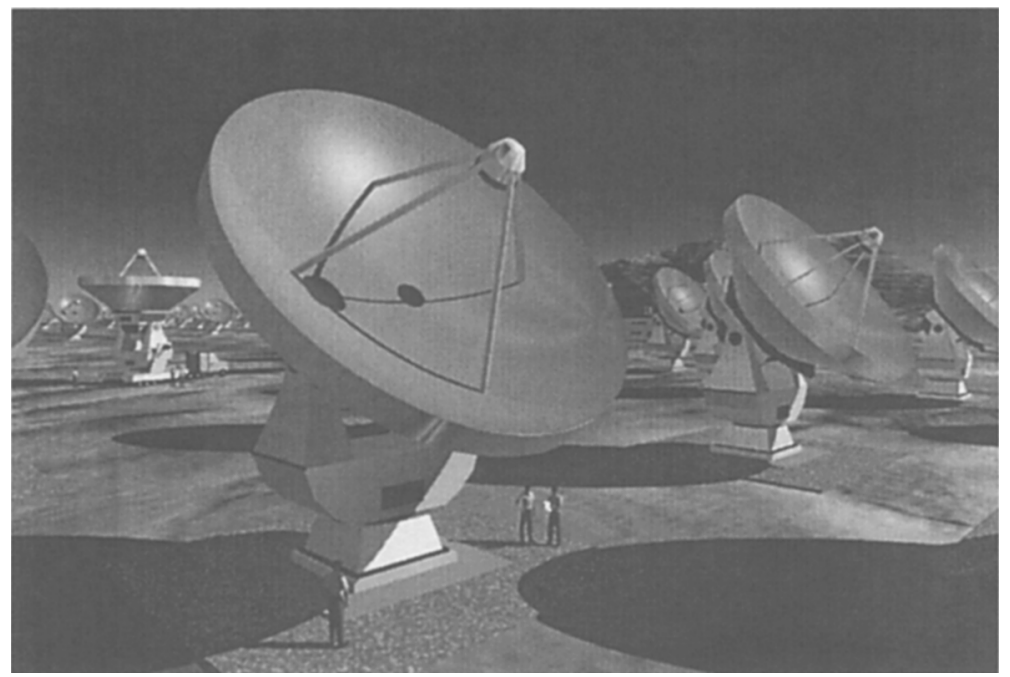

Figure 2. An artist's impression of several antennas of the Atacama Large Millimeter Array in a compact configuration.

Submillimeter Array (see Moran 1998), currently under construction on Mauna Kea, will consist of eight $6 \mathrm{~m}$ antennas. For an overview of millimeter interferometry, see the proceedings edited by Ishiguro \& Welch (1994).

\section{ALMA - The Future of (Sub)millimeter Interferometry}

It is clear that further progress in submillimeter astronomy requires an instrument capable of opening new vistas in wavelength coverage, spatial resolution, sensitivity, and mapping fidelity.

These demands will all be met by the Atacama Large Millimeter Array (ALMA), which is currently in a design and development phase as a joint project of European and American (US and Canadian) institutions. Japan is likely to become an equal partner in ALMA in the near future.

An excellent site on the Llano de Chajnantor in Chilean high Atacama desert near San Pedro de Atacama has been been identified for ALMA, which allows submillimeter operation most of the time and permits placement of antennas for optimized $u v$-coverage over an area of size several kilometers. Very diverse requirements for high surface brightness sensitivity on the one hand and high spatial resolution on the other will be met by relocating the array antennas in various configurations by means of transport vehicles. Longer baselines can be realized by moving antennas to adjoining areas. Fig. 2 gives an artist's impression of ALMA.

In Table 1, ALMA's basic parameters are presented. Its much larger collecting area, wider bandwidth, and the combination of advanced receiver technology and superior site result in a continuum sensitivity for point-like sources that is $\approx 100$ times higher than that of existing instruments. 


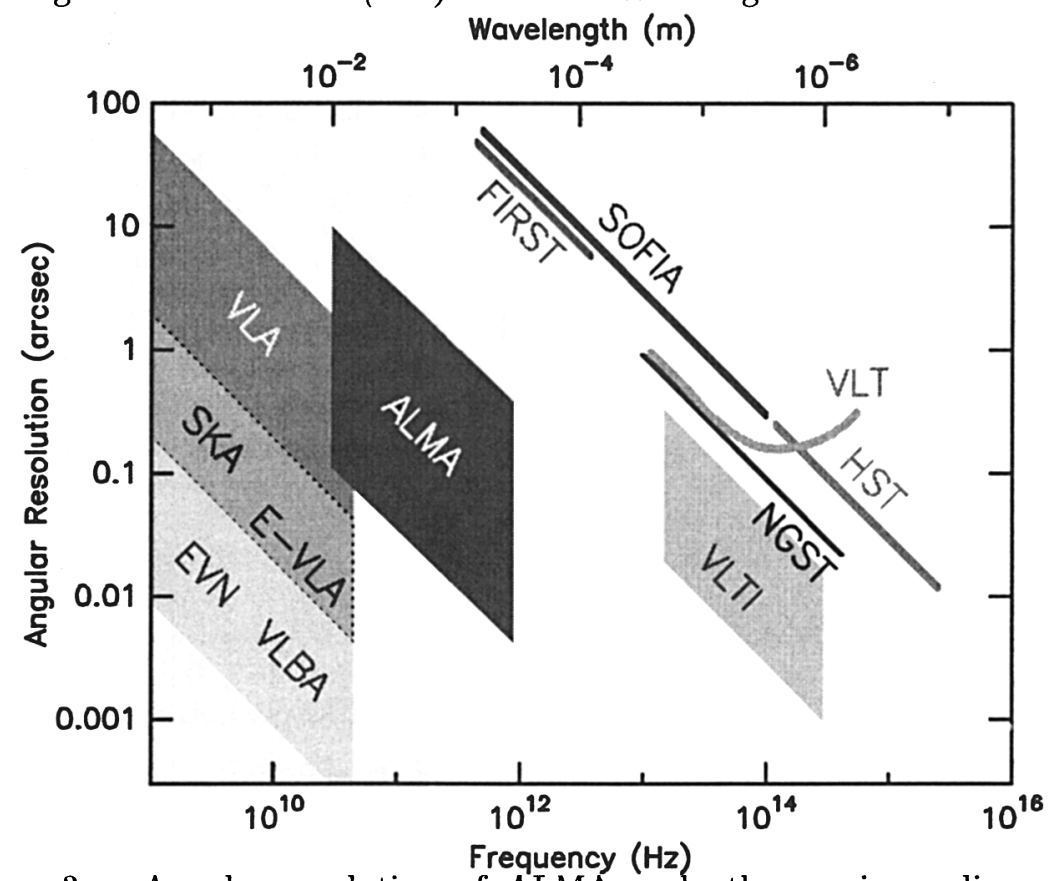

Figure 3. Angular resolution of ALMA and other major radio-, (sub)millimeter-, infrared-, and optical-wavelength facilities that are operating today or are expected to become available within the next ten years. Note that the Square Kilometer Array, which is currently being planned, will have resolution and wavelength coverage similar to the proposed Expanded Very Large Array (E-VLA) but a 20 times higher collecting area. Launch dates for the Far InfraRed Space Telescope (FIRST, now named Herschel Space Observatory) and the Next Generation Space Telescope (NGST) are 2007 and 2009, respectively, while the Stratospheric Observatory for Infrared Astronomy (SOFIA) will commence operations in 2002/2003. The development of the VLT Interferometer, i.e. the recently completed ESO Very Large Telescope in interferometry mode, is currently under way.

On the site selected for ALMA, long-range monitoring over a six year period has shown that excellent atmospheric conditions, i.e. less than $1 \mathrm{~mm}$ of precipitable water vapor, allowing operations in all of the submillimeter windows, prevail for $\approx 50 \%$ of the time. ALMA's antenna specifications call for an rms surface accuracy of $20 \mu \mathrm{m}$, allowing efficient submillimeter operation in all the atmospheric windows below $1 \mathrm{THz}$.

With ALMA, spatial resolutions better than 0."02 will be achieved, comparable to the diffraction limit of an 8 meter telescope at optical wavelengths (Fig. 3). ALMA's design considers the necessary requirements in signal transmission technology and allows for fast switching, which may be needed to achieve the highest angular resolutions. 
Table 1. Atacama Large Millimeter Array - Technical Summary

Array

Antennas

Total collecting area

$64 \times 12 \mathrm{~m}$ (rms surface accuracy $20 \mu \mathrm{m})$

Array layout

$7238 \mathrm{~m}^{2}$

Can be reconfigured from most compact

(150 m filled array) to highest resolution configuration with $12 \mathrm{~km}$ maximum antenna separation

Angular resolution

0 ". $2 \times \frac{\text { wavelength }(\mathrm{mm})}{\text { baseline }(\mathrm{km})}$

Receiver Bands ${ }^{a}$

$86-119 \mathrm{GHz}$

$211-275 \mathrm{GHz}$

$275-370 \mathrm{GHz}$

$602-720 \mathrm{GHz}$

Dual polarization

$T_{\mathrm{rx}}<6 \mathrm{~h} \nu / \mathrm{k} \mathrm{K} \mathrm{SSB}$

$T_{\mathrm{rx}}<6 \mathrm{~h} \nu / \mathrm{k} \mathrm{K} \mathrm{SSB}$

$T_{\mathrm{rx}}<4 \mathrm{~h} \nu / \mathrm{k} \mathrm{K}$ DSB

$T_{\mathrm{rx}}<5 \mathrm{~h} \nu / \mathrm{k} \mathrm{K} \mathrm{DSB}$

All frequency bands

\section{Correlator}

Correlated baselines

Bandwidth

2016

Spectral channels

$8 \mathrm{GHz}$ for each polarization 4096 per IF

\footnotetext{
${ }^{a}$ Highest priority receiver bands to be implemented for initial operation. In total, ten bands are needed to cover all atmospheric windows between 30 and $950 \mathrm{GHz}$ accessible from the ground. Single-sideband (SSB) and double-sideband (DSB) receiver noise temperatures, $T_{\mathrm{rx}}$, are listed in units of the "quantum limit" $\mathrm{h} \nu / \mathrm{k}$, where $\nu$ is the frequency.
}

ALMA's combination of collecting area, wide receiving bandwidth, excellent receivers and site will allow continuum imaging with sub-Kelvin brightness temperature sensitivity at resolutions of $0 . \prime 22$ or better.

Finally, ALMA has superb imaging capability. The 2016 baselines formed by its 64 antennas will provide very high dynamic range, even for instantaneous "snapshots" and permit rapid imaging.

With its sensitivity and resolving power, ALMA will allow ground-breaking observations relevant to all fields of astrophysics. (Sub)millimeter observations with ALMA will become important cornerstones of multi-wavelength studies, which for optimal scientific return require comparable resolution in the different wavelength regimes. As demonstrated in Fig. 3, ALMA's spatial resolution is in the same range as that of existing and future major telescopes from radio to optical wavelengths.

A comprehensive overview of ALMA's scientific potential can be found in the proceedings of the "Science with Large Millimetre Arrays" Workshop edited by Shaver (1996). Downes (1999) reviews recent results and future directions of (sub)millimeter interferometry and also discusses the fundamental technological specifications for ALMA and the main science drivers, while Menten (2000) gives examples of interesting science addressed by ALMA from various fields of as- 
tronomy. Many state-of-the-art results obtained with millimeter interferometers are found in the present proceedings.

Fig. 3 shows quite conspicuously that for the near future no prospects exist for attaining high (i.e. sub-arcsecond) resolution in the far-infrared regime, i.e. between 1 and $10 \mathrm{THz}$ (or $30-300 \mu \mathrm{m}$ ). Given the enormous astrophysical importance of this frequency range, in which about $98 \%$ of the post-Big Bang photons are emitted, producing half of the total luminosity in the Universe, development of far-infrared interferometry appears highly desirable.

While an far-infrared interferometer array could be operated on the lunar surface (see, e.g., Burke 1990), at this point a space-borne project seems more realistic. Leisawitz et al. (1999, 2000) present a possible concept for such a mission: The Submillimeter Probe of the Evolution of Cosmic Structure (SPECS) is a interferometer consisting of three cold telescopes of $3 \mathrm{~m}$ aperture each, flying in a triangular formation and forming a spatial and spectral Michelson interferometer with baselines up to $1 \mathrm{~km}$. The telescopes are movable, possibly along tethers, so that good $u v$-coverage can be achieved. At $\lambda=250 \mu \mathrm{m}$ a maximum spatial resolution of $0 . \prime 05$ is attained over a $14^{\prime}$ field of view.

\section{References}

Barvainis, R. 1999, in ASP Conf. Ser. Vol. 156, Highly Redshifted Radio Lines, ed. C. L. Carilli, S. J. E. Radford, K. M. Menten \& G. I. Langston (San Francisco: ASP), 39

Blain, A. W., Longair, M. S. 1993, MNRAS, 264, 509

Braine, J., Hughes, D. H. 1999, A\&A, 344, 779

Burke, B. F. 1990, Science, 250, 1365

De Jong, T. et al. 1984, ApJ, 278, L67

Downes, D. 1999, Rev. Mod. Astr., 12, 69

Franceschini, A., Toffolatti, L., Mazzei, P., Danese, L., \& De Zotti, G. 1991, A\&A, 89, 285

Hughes, D. H. et al. 1998, Nature, 394, 241

Ishiguro, M., Welch, W. J. 1994, (ed.) IAU Coll. 140, Astronomy with Millimeter and Submillimeter Wave Interferometry (San Francisco: ASP)

Leisawitz, D., et al. 1999, in The Physics and Chemistry of the Interstellar Medium, ed. V. Ossenkopf, J. Stutzki \& G. Winnewisser (Herdecke: GCA-Verlag), 454

Leisawitz, D., et al. 2000, Proc. SPIE, 4013, 36

Menten, K. M. 2000, in From Extrasolar Planets to Cosmology: The VLT Opening Symposium, ed. J. Bergeron \& A. Renzini (Berlin: Springer), 78

Moran, J. M. 1998, Proc. SPIE, 3357, 208

Omont, A., Petitjean, P., Guilloteau, S., McMahon, R. G., Solomon, P. M., \& Pecontal, E. 1996, Nature, 382, 428

Sanders, D. B., Mirabel, I. F. 1996, ARA\&A, 34, 749

Shaver, P. A. 1996, (ed.), Science with Large Millimetre Arrays - Proceedings of the ESO-IRAM-NFRA-Onsala Workshop (Berlin: Springer) 